\title{
Morbilidad materna severa y casi pérdidas en los extremos de la edad reproductiva: resultados de un estudio transver- sal multicéntrico nacional (1)
}

\author{
Oliveira FC, Jr., Surita FG, Pinto ESJL, Cecatti JG, Parpinelli MA, Haddad SM, Costa ML, \\ Pacagnella RC, Sousa MH, Souza JP; Brazilian Network for Surveillance of Severe Ma- \\ ternal Morbidity Study Group. Severe maternal morbidity and maternal near miss in the \\ extremes of reproductive age: results from a national cross- sectional multicenter study. \\ BMC Pregnancy Childbirth 2014; 14(77) doi: 10.1186/1471-2393-14-77.
}

Análisis Crítico: Claudio Vera P-G, MSc ${ }^{1}$, Jorge A. Carvajal Cabrera, $\mathrm{PhD}^{1}$.

${ }^{1}$ Unidad de Medicina Materno-Fetal, División de Obstetricia y Ginecología, Escuela de Medicina. Pontificia Universidad Católica de Chile.

\section{RESUMEN (1)}

Objetivo: Evaluar la morbilidad materna grave y "near miss" en niñas adolescentes y mujeres sobre los 35 años en la Red Brasilera de Vigilancia de Morbilidad Materna Grave, usando un conjunto de criterios estandarizados, comparado con mujeres de 20 a 34 años de edad. Métodos: Estudio transversal conducido en 27 unidades de referencia obstétrica en Brasil. Todas las embarazadas admitidas a estos centros por el periodo de un año de vigilancia prospectiva fueron tamizadas para identificar casos de muerte materna (MM), "near miss" (NM) y otras morbilidades maternas graves (MMG). Indicadores de morbilidad materna y mortalidad fueron evaluados para los tres grupos etarios, se compararon las características sociodemográficas, clínicas y obstétricas, eventos gestacionales y perinatales, causa principal de morbilidad y retrasos en la atención. Dos modelos de análisis múltiple fueron realizados para estimar la razón de prevalencia ajustada para factores que estuviesen independientemente asociados con la ocurrencia de eventos maternos graves $(\mathrm{EMG}=\mathrm{MNM}+\mathrm{MD})$. Resultados: En los casos de morbilidades maternas severas y MM, la proporción de niñas adolescentes y mujeres mayores fue de $17 \%$ de cada una. El riesgo de NM materno o muerte fue $25 \%$ mayor en las mujeres mayores. La razón de NM materno y razón de mortalidad materna aumentaron con la edad, pero estas razones fueron también elevadas en las adolescentes de
10 a 14 años, si bien los números absolutos fueron bajos. En el análisis multivariado, las jóvenes no fueron identificadas como un factor independiente de eventos maternos graves, mientras esto fue verdad para las de mayor edad (PR 1,25; 1,07-1,45). Conclusión: EMG fueron altos en mujeres bajo los 14 años de edad y se incrementan con la edad en mujeres brasileras embarazadas.

\section{A. Relevancia clínica de la investigación}

La relación entre la edad y la mortalidad materna ha sido ampliamente reconocida a nivel global. Las razones de mortalidad a lo largo de la edad materna distribuyen en forma de $\mathrm{J}$ con aumento en los extremos de la vida acentuándose a medida que avanza la edad $(2,3)$. Diferenciar los factores que contribuyen a esta distribución permite focalizar intervenciones en grupos de alto riesgo y promover los grupos de bajo riesgo. La posibilidad de la evaluación de los eventos maternos graves (EMG) junto con sus factores asociados de una manera estandarizada (4), en el contexto de un sistema de vigilancia de salud materna a nivel nacional en Brasil (5), constituye una excelente oportunidad de medir el impacto y entender las causas de la morbilidad materna grave en los extremos de la vida reproductiva. Esto aporta información relevante tanto a clínicos como a directores de programa en la toma de decisiones para el cuidado de la salud de la mujer. 


\section{B. El estudio (1)}

Diseño: Estudio transversal analítico que incluye la vigilancia de 27 centros de referencia obstétrica en todas las regiones geográficas de Brasil desde julio del 2009 a junio del 2010. Para identificarlos casos con condiciones de potencial amenaza vital materna, "near miss" y mortalidad materna se utilizaron los criterios OMS (6). Los autores definen morbilidad materna grave como la suma de los casos con condiciones de potencial amenaza vital, los casos con amenaza vital (near miss) y las muertes maternas. Así también, definen como evento o resultado materno grave a la suma de los casos de amenaza vital (near miss) y muerte materna. Para identificar 750 casos de amenaza vital materna los autores estimaron necesario evaluar 75000 partos bajo una incidencia teórica de 10 casos de amenaza vital por cada 1000 partos. La edad materna fue categorizada en 3 grupos 10 a 19 años, 20 a 34 años y 35 a 49 años. Con subgrupos de 10 a 14 y de 35 a 39 años. Se evaluaron los datos socio-demográficos, historia obstétrica, condiciones mórbidas previas, cuidado prenatal y complicaciones obstétricas. También se evaluaron los retrasos en la atención obstétrica con formularios estandarizados. Se realizó control de calidad de los datos ingresados junto con manuales para la estandarización de conceptos y procedimientos. Las unidades de muestreo correspondieron a los 27 centros de atención obstétrica con al menos 1000 partos anuales mientras que las unidades de análisis fueron las fichas de cada mujer hospitalizada con una condición de potencial amenaza vital. Dado que no se recolectó información de las madres sin complicaciones, los indicadores fueron estimados con los nacidos vivos informados para Brasil para el año 2009 para cada categoría de edad. Las diferencias de los factores socio-demográficos y clínicos se estimaron entre las categorías de edad de las pacientes afectadas.
Se realizó análisis de regresión de Poisson para identificar factores que se asociaran a peores resultados de manera independiente (condiciones de potencial amenaza vital vs. amenaza vital o muerte) las categoría de edad más extrema se compararon con el estrato de 20 a 34 años como categoría de referencia en diferentes modelos. Se estimó la razón de prevalencia ajustada por el efecto de conglomerado y el resto de los factores.

Resultados principales: Durante el periodo de estudio se identificaron 9.555 casos de condiciones de potencial amenaza vital, amenaza vital y muerte materna en 82.144 nacidos vivos. Las adolescentes fueron responsables de un $17,9 \%$ y las madres de 35 a más años fueron responsables, a su vez de un $17 \%$ de los casos. En el total de afectadas la razón de MM fue de 170 por 100.000 nv, con 5,5 casos de amenaza vital (NM) por cada muerte materna. La descripción de las razones de MM y de la relación de casos de NM por MM para las distintas categorías de edad se resume en la Tabla I. Entre las diferencias de factores socio-demográficos y clínicos entre las categorías de edad destaca que las adolescentes presentaron mayor proporción de origen diferente de blanco, menor escolaridad, carencia de pareja estable, bajo peso al nacer y multiparidad comparado con el grupo de referencia. Las madres de más de 35 años presentaron mayor proporción de baja escolaridad, pareja estable, obesidad, antecedentes de morbilidad (hipertensión, diabetes, enfermedad cardiaca, enfermedad tiroidea), historia de abortos previos y menor número de controles prenatales. Entre las principales causas de morbilidad grave destaca una menor proporción de causas hemorrágicas y mayor proporción de causas hipertensivas en mujeres adolescentes. Las adolescentes presentaron más situaciones definidas como retrasos para obtener adecuado cuidado obstétrico para la complicación.

Tabla I

RESUMEN DE RESULTADOS (1)

\begin{tabular}{lccccc}
\hline $\begin{array}{l}\text { Grupo } \\
\text { de edad }\end{array}$ & $\begin{array}{c}\text { Razón de } \\
\text { mortalidad materna } \\
\text { por 100.000 nv. }\end{array}$ & NM:MM & $\begin{array}{c}\text { NM por } \\
1.000 \mathrm{nv}\end{array}$ & $\begin{array}{c}\text { RMG } \\
\text { (NM + MM) } \\
\text { por } 1.000 \mathrm{nv}\end{array}$ & $\begin{array}{c}\text { Razón de prevalencia de } \\
\text { RMG } \\
(\text { IC 95\%) }\end{array}$ \\
\hline 10 a 14 años & 376,4 & $2,3: 1$ & 8,78 & 12,55 & $0,97(0,57$ a 1,65) \\
15 a 19 años & 121,9 & $5,8: 1$ & 7,06 & 8,27 & $0,88(0,76$ a 1,03) \\
20 a 34 años & 165,4 & $5,1: 1$ & 8,39 & 10,05 & Referencia \\
35 a 39 años & 230,6 & $7,4: 1$ & 17,52 & 19,83 & $1,19(1,00$ a 1,41$)$ \\
40 a 49 años & 440,8 & $7,1: 1$ & 31,40 & 35,81 & $1,52(1,19$ a 1,93) \\
\hline
\end{tabular}

$\mathrm{NM}=$ Near Miss o casos de amenaza vital materna, $\mathrm{MM}=$ mortalidad materna, $\mathrm{RMG}=$ Resultado Materno Grave (suma de casos de amenaza vital y muertes maternas). 
Análisis multivariado entre las adolescentes y categoría de referencia detectó que las mujeres con infección, condiciones médico-quirúrgicas, sangrado y las que tuvieron retraso en la atención tuvieron mayor riesgo de un resultado materno grave (NM o $\mathrm{MM})$. El riesgo también fue mayor en presencia de condiciones clínicas como diabetes, cáncer y otras en mujeres de origen diferente de blanco. La obesidad se asoció a un $50 \%$ de reducción en el riesgo de NM y MM. La edad materna no fue un factor condicionante de mayor riesgo.

Análisis multivariado entre las mujeres de 35 o más años y la categoría de referencia también mostró un aumento del riesgo al tener infección, condiciones médico-quirúrgicas, sangrado, y si tenían retraso en la atención. Entre las condiciones médicas preexistentes destacaron enfermedad renal, diabetes, talasemia o anemia de células falciformes, adicción a drogas, control prenatal en otro servicio. Pareja estable y obesidad se mostraron como factores protectores. La edad materna aumentó significativamente el riesgo de resultado materno grave en un $25 \%$.

\section{Análisis crítico}

Estudio transversal orientado a determinar factores pronósticos de distintas categorías de edad de mujeres que presentaron condiciones de potencial amenaza vital, amenaza vital y muerte materna de acuerdo con los criterios definidos por OMS para la detección y clasificación de resultados maternos graves (NM y MM).

Riesgo de sesgo: Realizamos la evaluación del riesgo de sesgo de acuerdo con la guía de análisis de estudios sobre pronóstico (7) para lo cual indagamos información sobre la metodología descrita previamente $(5,8)$. La Red Brasilera de Vigilancia de Morbilidad Materna Grave se constituyó a partir de centros de atención obstétrica con más de 1.000 partos anuales que voluntariamente aceptaron participar de las 5 regiones geográficas de Brasil con el objetivo de validar los criterios OMS para clasificar las condiciones de potencial amenaza vital, amenaza vital y muerte materna. Así se incluyeron 9.555 casos maternos mientras ocurrieron 82.144 nacidos vivos en los centros incluidos en el periodo planificado. Constituyendo una muestra del 3\% de los nacidos vivos en Brasil estimados para el año 2009. Dado que la inclusión de los centros es voluntaria y que no se planificó un muestreo aleatorio de centros en las diferentes regiones, consideramos que la representatividad de la diversidad de morbi-mortalidad materna para Brasil es limitada. Por otro lado, cumple apropiadamente con el objetivo planificado de representar el espectro de la morbilidad y mortalidad materna para los objetivos de la validación de los instrumentos, así los casos tienen una razón de MM estimada en 170 por 100.000 nv, superior a la razón de MM estimada para Brasil en el año 2009 de 73,1 por 100.000 nv. No es posible determinar si la distribución de las categorías de edad materna representa a la distribución de los quinquenios en las madres de Brasil o de los centros incluidos, la información disponible sobre la distribución de las edades maternas de los nacidos vivos en Brasil para el año 2009 (http://tabnet.datasus.gov.br/cgi/deftohtm.exe?sinasc/cnv/nvuf.def) muestra que las madres de 10 a 19 años representan el $20 \%$ del total, las de 20 a 34 años el $70 \%$ y las de 35 a 49 años el 10\%, cifras diferentes a la distribución encontrada en los casos de morbilidad materna. Con respecto a los criterios de inclusión se restringe a las pacientes hospitalizadas, lo cual no considera el seguimiento de pacientes hasta los 42 días postparto ni las MM ocurridas fuera del contexto de un centro de atención.

La principal fortaleza del estudio es la aplicación de sistemática de los criterios de clasificación OMS para las condiciones de potencial amenaza vital y de amenaza vital (near miss) estos criterios basados en la disfunción de órganos han demostrado muy buena correlación con otro método de evaluación más detallada de la disfunción de órganos, así como elevada exactitud para la detección de casos de muerte materna (9).

Las estimaciones consideran una serie de factores confundentes y estima la contribución independiente tanto de factores socio-económicos como clínicos, entregando información relevante para la focalización de intervenciones y recursos. Llama la atención de que la obesidad resulta factor protector de resultado materno grave, tanto para la evaluación de adolescentes como las mayores de 35 al compararlas con el grupo control, a diferencia de otros estudios que la identifican como factor de riesgo para morbilidad materna grave $(10,11)$, los autores destacan que no se conocía el peso en una proporción importante de las pacientes del estudio lo cual puede afectar la estimación, por otro lado puede ser un factor de riesgo emergente en la categoría seleccionada como control, por lo cual los resultados deben ser considerados con cautela. Por otro lado, el diseño presentado permite el estudio de la contribución independiente de los factores en su aspecto pronóstico ya que todas las pacientes incluidas son casos de "morbilidad materna grave". Con respecto a los resultados los autores muestran que los extremos de la edad materna constituyen factores pronósticos cada vez que una mujer embarazada presenta una condición tan o más grave que una potencial amenaza vital bajo los criterios OMS.

Comentarios: Los autores consideran morbilidad materna grave como término para describir la suma de casos de potencial amenaza vital, casos de amenaza vital (NM) y muertes maternas. Sin embargo, dado que "near miss" no cuenta con una traducción 
directa del concepto al español la recomendación del Centro Latinoamericano de Perinatología en conjunto con la Organización Panamericana de la Salud es de reservar el término de morbilidad materna grave a los casos donde una mujer casi muere, pero sobrevive a una complicación que ocurre durante el embarazo, el parto o dentro de los 42 días de terminado el embarazo (12), definición que corresponde al término "near miss" o amenaza vital materna de la iniciativa OMS (4).

Conclusión: Estudio observacional con moderado a alto riesgo de sesgo que estima el impacto pronóstico de categorías de edad materna de pacientes con condiciones de amenaza vital sobre el riesgo de amenaza vital y muerte materna, así como la contribución independientes de otros factores en las categorías de edad materna extrema en un estudio transversal. Muestra que a medida que aumenta la edad materna de las madres afectadas por condiciones de potencial amenaza vital aumenta el riesgo de mortalidad materna y el riesgo de casos de amenaza vital o near miss. La mayoría de estas estimaciones confirman diferencias observadas entre las categorías de edad materna para mortalidad materna ampliándolas a la morbilidad materna grave, pero son difíciles de transferir directamente a otros contextos dadas las limitaciones del estudio. Estudios prospectivos con menor riesgo de sesgo y apropiado seguimiento son necesarios para verificar y mejorar la confianza en las estimaciones.

\section{REFERENCIAS}

1. Oliveira FC, Jr., Surita FG, Pinto ESJL, Cecatti JG, Parpinelli MA, Haddad SM, et al. Severe maternal morbidity and maternal near miss in the extremes of reproductive age: results from a national cross- sectional multicenter study. BMC Pregnancy Childbirth 2014; 14(77) doi: 10.1186/1471-2393-14-77.

2. Kassebaum NJ, Bertozzi-Villa A, Coggeshall MS, Shackelford KA, Steiner C, Heuton KR, et al. Global, regional, and national levels and causes of maternal mortality during 1990-2013: a systematic analysis for the Global Burden of Disease Study 2013. Lancet. 2014;384(9947):980-1004.

3. Donoso E, Carvajal JA, Vera C, Poblete JA. La edad de la mujer como factor de riesgo de mortalidad materna, fetal, neonatal e infantil. Rev Méd Chil 2014;142:168-174.

4. World Health Organization. Evaluating the quality of care for severe pregnancy complications: the WHO near-miss approach for maternal health. 2011. Disponible en: http://www.who.int/reproductivehealth/ publications/monitoring/9789241502221/en/. Acceso: 13 de marzo 2014.

5. Cecatti JG, Souza JP, Parpinelli MA, Haddad SM, Camargo RS, Pacagnella RC, et al. Brazilian network for the surveillance of maternal potentially life threatening morbidity and maternal near-miss and a multidimensional evaluation of their long term consequences. Reprod Health 2009;6:15.

6. Say L, Souza JP, Pattinson RC, WHO. working group on Maternal Mortality and Morbidity classifications. Maternal near miss--towards a standard tool for monitoring quality of maternal health care. Best Pract Res Clin Obstet Gynaecol 2009; 23(3):287-96.

7. Randolph A, Cook DI, Guyatt G. Prognosis. En: Guyatt $G$, Rennie $D$, Meade MO, Cook DI, editores. User's Guides to the Medical Literature: A Manual for Evidence-Based Clinical Practice. New York: McGraw Hill Medical; 2008.

8. Haddad SM, Cecatti JG, Parpinelli MA, Souza JP, Costa ML, Sousa MH, et al. From planning to practice: building the national network for the Surveillance of Severe Maternal Morbidity. BMC Public Health 2011;11:283.

9. Souza JP, Gulmezoglu AM, Vogel J, Carroli G, Lumbiganon $\mathrm{P}$, Qureshi Z, et al. Moving beyond essential interventions for reduction of maternal mortality (the WHO Multicountry Survey on Maternal and Newborn Health): a cross-sectional study. Lancet 2013;381(9879):1747-55.

10. Gray KE, Wallace ER, Nelson KR, Reed SD, Schiff MA. Population-based study of risk factors for severe maternal morbidity. Paediatr Perinat Epidemiol 2012;26(6):506-14.

11. Zwart JJ, Richters JM, Ory F, de Vries JI, Bloemenkamp KW, van Roosmalen J. Severe maternal morbidity during pregnancy, delivery and puerperium in the Netherlands: a nationwide population-based study of 371,000 pregnancies. BJOG 2008;115(7):84250.

12. Centro Latinoamericano de Perinatología. Plan de acción para acelerar la reducción de la mortalidad materna y morbilidad materna grave: Estrategia de Monitoreo y Evaluación. 2012. Organización Panamericana de la Salud/ Organización Mundial de la Salud. Disponible en: http://www.paho.org/clap/index. php?option=com_content\&view=article\&id=173:estra tegia-de-monitoreo-y-evaluacion-plan-reduccion-morbimortalidad-materna-grave\&catid=667: publicaciones \&ltemid=234. Acceso el 13 de Marzo de 2014. 